\title{
Article
}

\section{Disease-dependent interaction policies to support health and economic outcomes during the COVID- 19 epidemic}

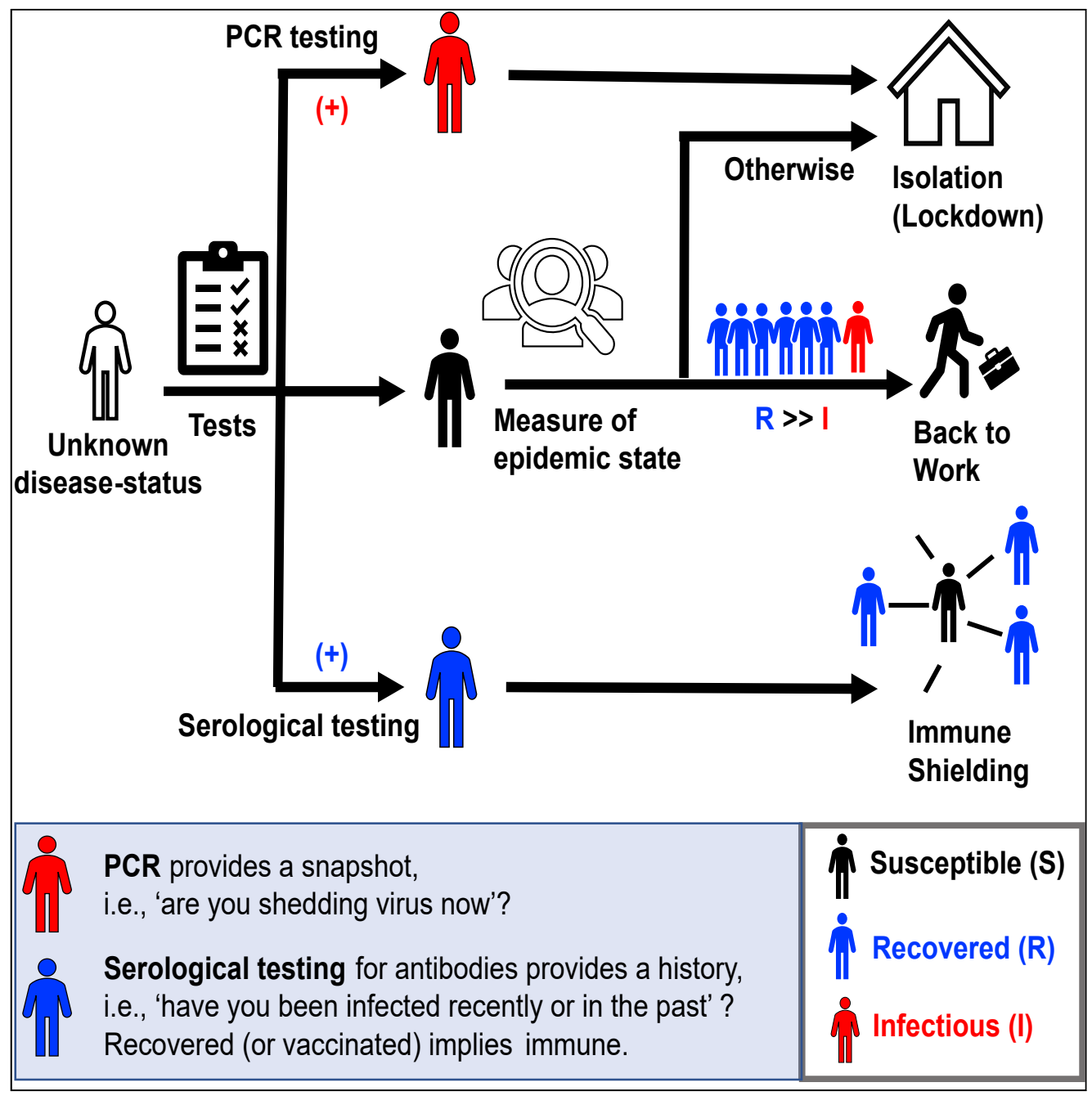

Guanlin Li,

Shashwat Shivam,

Michael E.

Hochberg, Yorai

Wardi, Joshua S.

Weitz

jsweitz@gatech.edu

Highlights

We develop a feedback approach to mitigate COVID-19 and enable

safer return-to-work

Feedback policies show greater robustness to uncertainty compared to optimal control

Return-to-work is accelerated by large-scale viral and serological testing

Large-scale testing guides targeted changes in behavior rather than enmasse lockdowns 


\title{
Article
}

\section{Disease-dependent interaction policies to support health and economic outcomes during the COVID-19 epidemic}

\author{
Guanlin Li, 1,2,7 Shashwat Shivam, 3,7 Michael E. Hochberg, ${ }^{4,5}$ Yorai Wardi, ${ }^{3}$ and Joshua S. Weitz 2,6,8,*
}

\begin{abstract}
SUMMARY
Lockdowns and stay-at-home orders have partially mitigated the spread of Covid19. However, en masse mitigation has come with substantial socioeconomic costs. In this paper, we demonstrate how individualized policies based on disease status can reduce transmission risk while minimizing impacts on economic outcomes. We design feedback control policies informed by optimal control solutions to modulate interaction rates of individuals based on the epidemic state. We identify personalized interaction rates such that recovered/immune individuals elevate their interactions and susceptible individuals remain at home before returning to pre-lockdown levels. As we show, feedback control policies can yield similar population-wide infection rates to total shutdown but with significantly lower economic costs and with greater robustness to uncertainty compared to optimal control policies. Our analysis shows that test-driven improvements in isolation efficiency of infectious individuals can inform disease-dependent interaction policies that mitigate transmission while enhancing the return of individuals to pre-pandemic economic activity.
\end{abstract}

\section{INTRODUCTION}

As of 7 March 2021, more than 116,166,652 cases of coronavirus disease 2019 (COVID-19) have been reported worldwide with more than 2,582,528 deaths globally (World Health Organization, 2021). Starting at the reported origin of the pandemic in Wuhan, China, control measures have been implemented in most countries where outbreaks have occurred (Flaxman et al., 2020; Chinazzi et al., 2020; Wells et al., 2020; Cowling et al., 2020; Ferguson et al., 2020; Kraemer et al., 2020). Multiple public health strategies are being deployed to slow outbreaks, and although recommendations always include social distancing and isolation of confirmed cases, the full spectrum of measures and levels of adherence differ from country to country, making assessments of strategy efficacy difficult (see (Flaxman et al., 2020), controversy surrounding (Zhang et al., 2020)).

The non-pharmaceutical control strategies for COVID-19 largely follow those employed in previous viral epidemics, including SARS, Ebola and MERS. Initial strategies can be broadly grouped into mitigation and suppression, where the former attempts to preserve essential health care services and contain morbidity and mortality, whereas the latter imposes more severe, emergency restrictions to prevent health care system collapse and provide conditions for easing-off toward less intense mitigation strategies (Walker et al., 2020). Both mitigation and suppression approaches carry considerable social and economic costs, meaning that policymakers and the public at large only adopt them for short time periods (OECDa, 2020). A problem is that control measures have often been applied irrespective of an individual's disease status (and/or likely infection risk severity) and are driven, in part, by the absence of information-driven alternatives.

Hence, distinct from lockdowns, there is an increasing interest in implementing population-wide prevention methods that decrease transmission risk while enabling economic re-engagement. Examples of such measures include mask-wearing (Greenhalgh et al., 2020; Chu et al., 2020), contact-free interactions (Brynjolfsson et al., 2020), and restructuring of physical spaces (Bodkin et al., 2020). The use of masks, in particular, has been shown to be effective at reducing respiratory transmission of SARS-CoV-2, particularly

\footnotetext{
${ }^{1}$ Interdisciplinary Graduate Program in Quantitative Biosciences, Georgia Institute of Technology, Atlanta, GA, USA

${ }^{2}$ School of Physics, Georgia Institute of Technology, Atlanta, GA, USA

${ }^{3}$ School of Electrical and Computer Engineering, Georgia Institute of Technology, Atlanta, GA, USA

4ISEM, Université de Montpellier, CNRS, IRD, EPHE, Montpellier, France ${ }^{5}$ Santa Fe Institute, Santa Fe, NM, USA

${ }^{6}$ School of Biological Sciences, Georgia Institute of Technology, Atlanta, GA, USA

${ }^{7}$ These authors contributed equally

${ }^{8}$ Lead contact

*Correspondence: jsweitz@gatech.edu

https://doi.org/10.1016/j.isci. 2021.102710
} 
when individuals in a potentially infectious interaction routinely wear them (Feng et al., 2020; Cheng et al., 2020). These population-wide measures still carry uncertainty since individuals are expected to behave uniformly irrespective of their disease status. COVID-19 screening provides a complementary route, and despite costs may confer both health and economic benefit (Atkeson et al., 2020) to overcome the negative impact on the economy due to pandemic-related shutdowns (Baqaee et al., 2020). As the scale of COVID19 testing has increased, jurisdictions may also have an opportunity to consider implementing tactical mitigation strategies informed by testing.

Testing for infected status can, in theory, be used to initiate targeted isolation, identification and tracing of contacts, quarantining of contacts, and then selected testing of contacts (Ferretti et al., 2020). If done rapidly and at scale, this kind of targeted PCR-based testing can provide early detection of cases and help break new chains of transmission (He et al., 2020; Yong et al., 2020; Gibson et al., 2021; Denny, 2020; Specht et al., 2021). The effectiveness of contact-tracing based control strategies hinges on the accurate identification and isolation of exposed and infectious cases. Intensive and stringent testing and isolation policies have even enabled some countries to reopen (Coglianese and Mahboubi, 2021). Slow return of test results (primarily) and false negatives (as a secondary factor) limit the effectiveness of testbased control policy (Larremore et al., 2021). A complementary tactic is the strategic deployment of immune individuals to effectively dilute transmission events (Kraay et al., 2020; Weitz et al., 2020a). Similar to the identification of infected individuals through PCR tests, immunity to SARS-CoV-2 is assessed through serological testing for protective antibodies (Amanat et al., 2020; Krammer and Simon, 2020) or presumed through vaccination. The overall benefits of shielding, as quantified via detailed modeling studies, have been shown to outweigh the potential costs associated with false positives insofar as high-quality tests are utilized (Kraay et al., 2020). Population-wide interventions, testing efforts, the selective confinement or deployment of people contingent on their infection status, and inaccuracies and limited adherence to policies, combine to create a challenging landscape for the persistent control of outbreaks (Fauci et al., 2020).

Non-pharmaceutical COVID-19 control until effective vaccines become widely available will necessarily involve periods of reduced social and economic activity; i.e., 'business, but not as usual'. Control efforts are already generating hardship and could in the longer-term result in social unrest and increased mortality (OECD, 2020b; Douglas et al., 2020; Hsiang et al., 2020). Here we confront a joint problem: how to identify policies that aim to reduce fatalities arising from COVID-19 while also enabling economic engagement. First, we use optimal control to assess both health and economic outcomes in an susceptible exposed infectious recovered (SEIR) disease model framework. There is a substantial and growing literature on optimal control for COVID-19, the bulk of which focuses on non-personalized release policies or policies that target age- or risk-stratified groups (Bonnans and Gianatti, 2020; Flaxman et al., 2020; Ferguson et al., 2020; Walker et al., 2020; Morris et al., 2021; Zhao and Feng, 2020). Here, we identify optimal control policies to modulate interaction rates based on disease - unifying prior efforts centered on isolation and shield immunity. We find that intermediate policy outcomes can do nearly, as well as strict public health scenarios, without incurring the severe costs as suppression-centered policies. However, optimal controls can be fragile, when applied in practice given that they rely on time-rather than state-based interventions; the consequence of mistiming interventions can be severe (Morris et al., 2021), Hence, guided by the optimal control analysis, we identify state-dependent policies similar to feedback control that provide actionable guidance for individual behavior. As we show, using population-wide PCR testing for infection alongside immune status can reduce COVID-19 transmission while enabling more individuals to return-towork sooner and with fewer restrictions than would otherwise be possible.

\section{RESULTS}

Optimal control framework for state-dependent contact rates policies that balance public health and socioeconomic costs

We develop an optimal control framework to identify policies that address the tension between decreasing contacts (that reduce new infections) with increasing contacts (that are linked to socioeconomic benefits). We represent the epidemic using a SEIR nonlinear dynamic model (see supplemental information for complete details; see Figure 1). In doing so, the force of infection is influenced by state-specific contact rates $C_{S}$, $C_{E}, C_{l}$ and $C_{R}$ for susceptible, exposed, infectious and recovered/immune individuals, respectively - these different levels form the basis for a control policy that directs individuals to interact at different levels depending on their test status. 


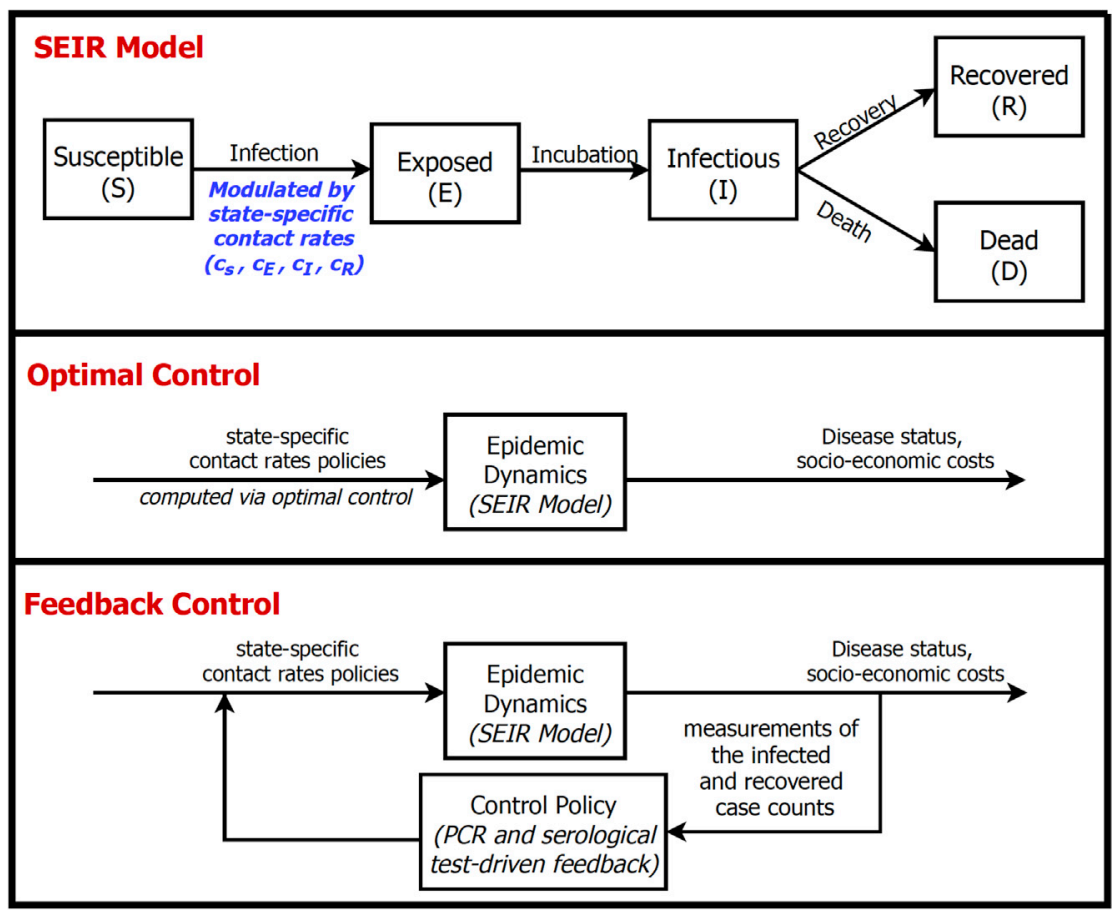

Figure 1. Epidemic dynamics with optimal and feedback control of disease status-driven contact rates (Top) SEIR model schematic in which the force of infection is modulated by state-specific contact rates, see text and supplemental information for details. (Middle) Diagram of optimal control approach: contact rates are pre-specified given model structure and estimate of parameters and current conditions. (Bottom) Diagram of feedback control approach: contact rates are updated in real-time based on measurements of the infected and recovered/immune case counts via testing surveillance.

In the optimal control framework, a set of state-specific contact rates are identified that minimize the appropriately weighted sum of what we term 'public health' and 'socioeconomic' costs. Public health costs are quantified both by average infected levels and cumulative deaths. Socioeconomic costs are quantified in terms of reductions in the total rate of interactions and by shifts in state-specific contact rates. The optimal control 'solution' is then a time-dependent set of disease-specific rates which are both shaped by and shape the epidemic itself (see supplemental information for details on the gradient projection algorithm used to identify the solution). Note that we constrain the contact rate of exposed individuals to be equal to that of susceptible individuals given the challenges of timely identification of exposed individuals who are not yet infectious (and presumably have insufficient viral titer to be identified using screening tests; an issue we return to in the discussion). Finally, we utilize the parameter $\xi$ to regulate the relative importance of costs associated with death and spread of infection vs. socioeconomic impact.

Figure 2 shows the results of comparing a baseline outbreak (i.e., neglecting public health costs, given weighting parameter $\xi=0$ ) to a full lockdown scenario (i.e., neglecting socioeconomic costs with $75 \%$ isolation for all, $\xi>>1$ ) and a balanced scenario with optimized contact rates (i.e., corresponding to $\xi=1$ ). At the time intervention starts (without any intervention in first 60 days), the outbreak has an ongoing incidence of 19 (per 100,000) per day, prevalence of $0.4 \%$, and a cumulative infection level of $0.8 \%$. As shown in Figure 2 , in the baseline scenario, the disease spreads through the population leading to $94 \%$ cumulative infection (as expected given strength-size relationships for $\mathcal{R}_{0}=3$ ). In contrast, a full lockdown scenario with $75 \%$ reduction in contact rates of all individuals after 60 days leads to a total outbreak size of $4 \%$ of the population. The optimal control solution in the balanced case $\xi=1$ reveals a potential route to jointly address public health and socioeconomic cost. From the perspective of public health, the optimal control solution leads to $25 \%$ cumulative infections. In addition, the socioeconomic costs in the optimal control case are higher in the short-term but approach that of the baseline scenario in the long-term. Indeed, the effective reproduction number identified via an optimal control framework in the balanced scenario gradually reduces to sub-critical levels (close to an effective reproduction number, $\mathcal{R}_{\text {eff }}=0.75$ ) while gradually relaxing 
A

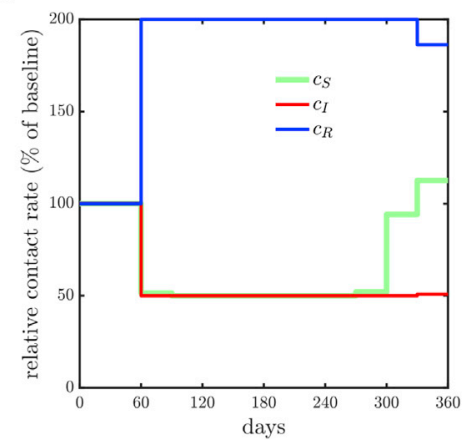

B

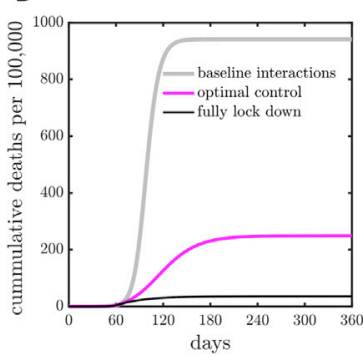

C

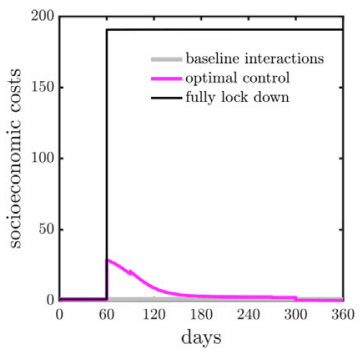

D

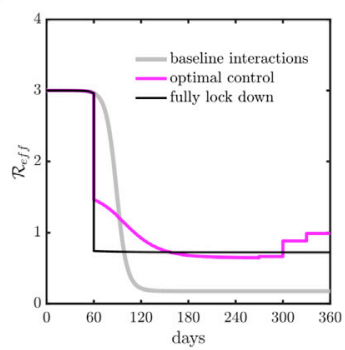

Figure 2. Health and economic outcomes associated with COVID-19 dynamics

Comparison of health and economic outcomes of COVID-19 given various interventions: baseline interactions (i.e., no intervention); optimal contact rate intervention (balance both health and economic outcomes) and fully lock down intervention (applied to all the subpopulations) with 75\% isolation efficiency.

(A) The optimal contact rate relative to the baseline contact rate (denoted as $100 \%$ ) with $50 \%$ isolation effectiveness and shield immunity level 2

(B) Cumulative deaths (health outcome) during the epidemic.

(C) Socioeconomic costs (economic outcome) during the epidemic.

(D) Measure of effective reproduction number $\left(\mathcal{R}_{\text {eff }}\right)$ for different interventions during the epidemic.

controls over time. The optimal control solutions are shown in Figure 2A. The optimal control solutions differ based on disease status, recovered/immune individuals elevate their interactions, infectious individuals isolate, and susceptible individuals lockdown before gradually returning to pre-lockdown levels.

\section{Personalized, test-based optimal control policies and their impact on public health and socioeconomic outcomes}

In order to explore the mechanisms identified by the optimal control framework, we systematically modulate the effectiveness of isolation and evaluate its effect on the state-dependent optimal contact rates and disease dynamics. In practice, isolation effectiveness is influenced by availability, accuracy, and speed of testing, as well as fundamental limitations on an individual's ability to isolate (which can vary with socioeconomic and other factors). Figures 3A-3C evaluate low, medium, and high efficiency of isolation spanning $25 \%, 50 \%$, and $75 \%$ reduction in the contact rates of infected individuals, respectively. As is evident, the optimal control solutions for state-dependent contact rates vary significantly with isolation effectiveness; suggesting that COVID-19 response policies that can vary with disease status may open up new possibilities to balance public health and socioeconomic outcomes.

First, in the low (25\%) or medium (50\%) effectiveness cases, susceptible, exposed, and infectious individuals adopt the maximal level of isolation. Inefficient isolation of infectious individuals elevates risks of new transmission that are not outweighed by socioeconomic benefits. Notably, the optimal control solution includes an elevated level of interaction by recovered/immune individuals. This finding recapitulates 'shield immunity' (Kraay et al., 2020; Weitz et al., 2020a), insofar as recovered/immune individuals are protected from reinfection over the course of the intervention. The elevated contacts of recovered/immune individuals have multiple effects: both diluting interactions by susceptibles (and reducing transmission risk) and by increasing socioeconomic activity. In contrast, for sufficiently high levels of isolation efficiency (75\%), the optimal control solutions suggest there is no need for a general lockdown. Instead, the combination of 
A
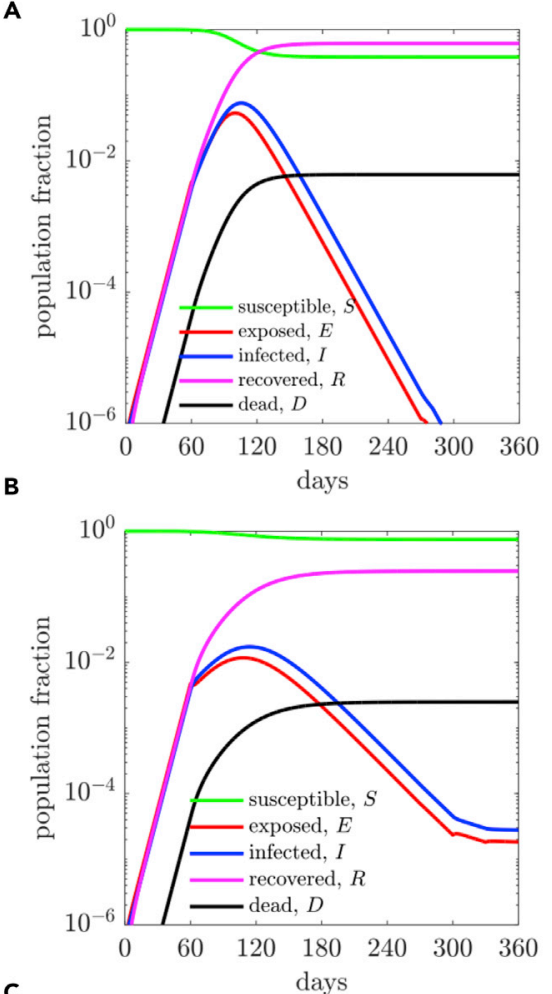

C

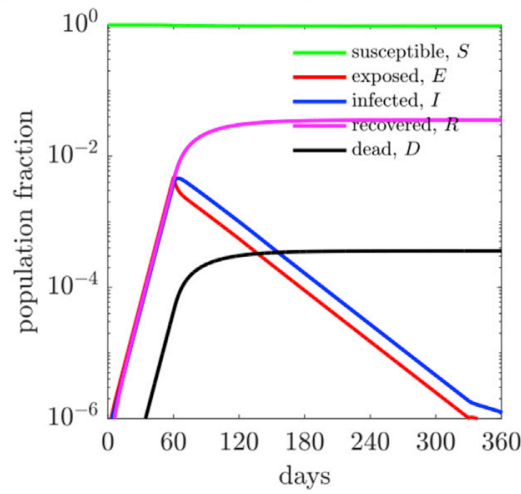

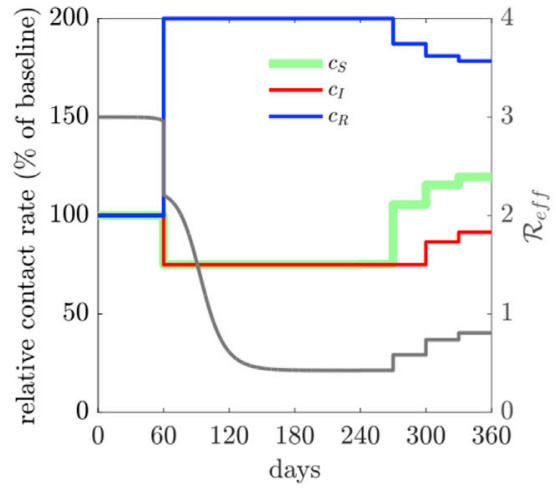
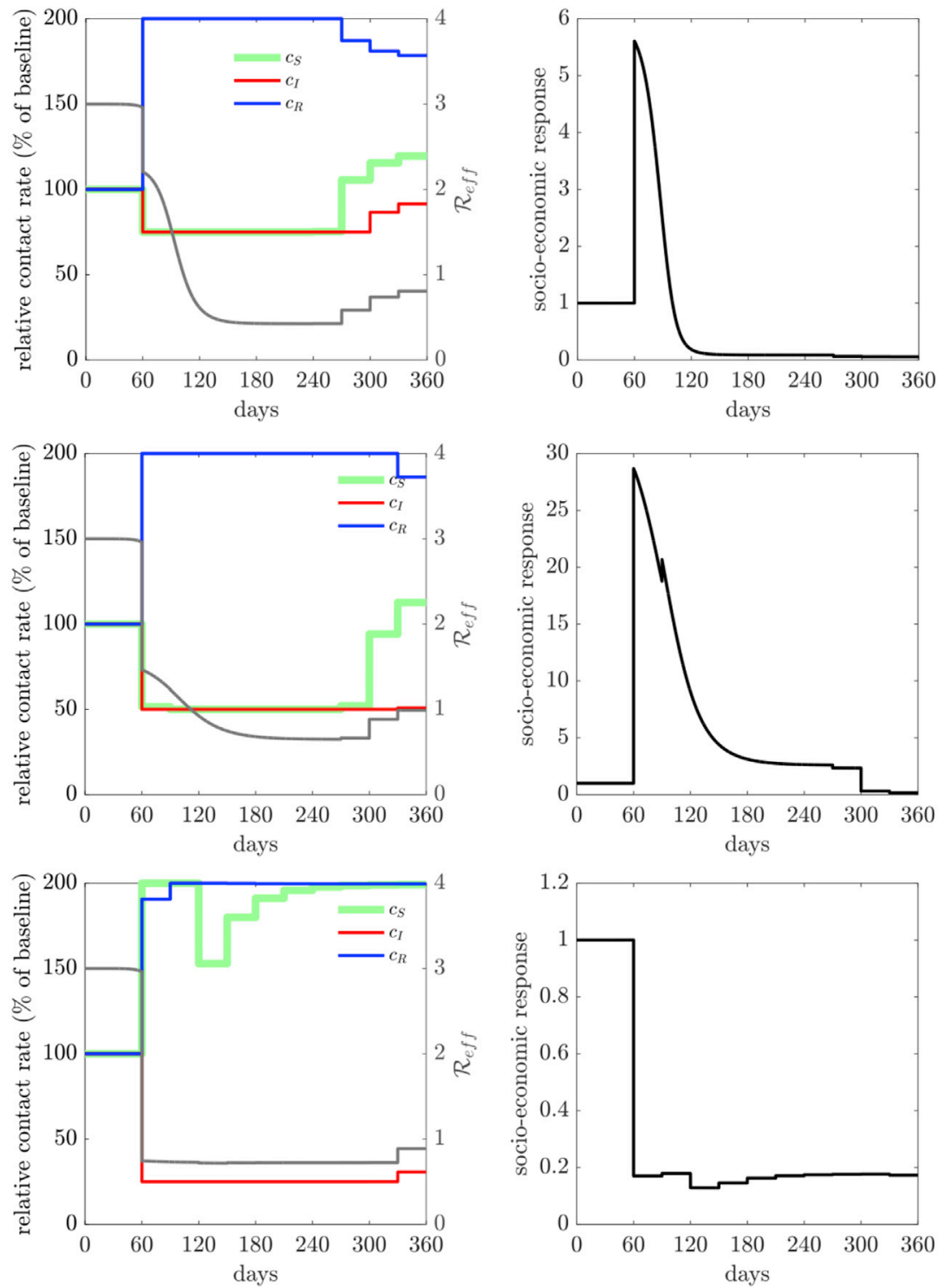

Figure 3. Epidemic dynamics and socioeconomic response with optimal contact rate interventions

SEIR dynamics with contact rate interventions for various isolation efficiencies, (A) $25 \%$ isolation efficiency; (B) $50 \%$ isolation efficiency and (C) $75 \%$ isolation efficiency. The relative importance ( $($ ) is 1 for all the cases (A), (B), and (C). The contact rate interventions start at 60 days, and people follow baseline (or normal) interactions before that. For all the isolation efficiency scenarios (three rows), the left panel shows the population dynamics given the optimal contact rate (related to the baseline contact rate, with $100 \%$ as baseline) shown in the middle panel. The gray curve in the middle panel represents the measure of corresponding effective reproduction number $\left(\mathcal{R}_{\text {eff }}\right)$. The right panel shows the corresponding socioeconomic costs. See supplemental information for additional scenarios.

infected case isolation and shielding by the subpopulation of recovered/immune individuals is sufficient to rapidly reduce and contain $\mathcal{R}_{\text {eff }}$ below 1 , leading to a decreasing number of new infections. We note that irrespective of isolation effectiveness, balancing public health and economic outcomes drives $\mathcal{R}_{\text {eff }}$ below 1 , but not necessarily to 0 (albeit, given the constraints imposed by lockdown efficiency, such an extreme reduction may not even be possible), and eventually increased immunity permits an easing-off in restrictions yielding an increase in $\mathcal{R}_{\text {eff }}$ (Hochberg, 2020).

\section{Sensitivity of optimal control approach to mistimed implementation of policies}

Despite its potential to balance public health and socioeconomic costs, a central drawback of optimal control solutions is the potential exponential growth of errors. Given the fact the COVID-19 dynamics are only 


\begin{tabular}{|c|c|c|c|c|}
\hline \multirow{2}{*}{$\begin{array}{l}\text { Optimal control approach } \\
\text { Efficiency of } \\
\text { isolation } \\
\text { (shielding }=2 \text { ) }\end{array}$} & \multicolumn{2}{|l|}{ Without delay } & \multicolumn{2}{|l|}{ With delay } \\
\hline & $\begin{array}{l}\text { Total deaths } \\
\text { (per } 100,000 \\
\text { individuals) }\end{array}$ & $\begin{array}{l}\text { Working } \\
\text { fraction }\end{array}$ & $\begin{array}{l}\text { Total deaths } \\
\text { (per } 100,000 \\
\text { individuals) }\end{array}$ & $\begin{array}{l}\text { Working } \\
\text { fraction }\end{array}$ \\
\hline $25 \%$ & 600 & $90.95 \%$ & 720 & $94.96 \%$ \\
\hline $50 \%$ & 250 & $67.13 \%$ & 510 & $83.65 \%$ \\
\hline $75 \%$ & 40 & $99.92 \%$ & 40 & $99.95 \%$ \\
\hline
\end{tabular}

The comparisons are made for isolation efficiencies of $25 \%, 50 \%$ and $75 \%$, with performance metrics of total deaths (per 100,000 individuals) and working fraction. The total death is significantly higher for the system with delay when the isolation efficiency is not $75 \%$, suggesting poor robustness to delay. Feedback control policy for balancing public health and socioeconomic costs.

partially observed (with significant uncertainty in the actual state), application of a policy requires an estimate of the time since epidemic initiation, what we term 'epidemic age'. In order to evaluate the sensitivity of optimal control policies due to mistiming, we first computed the optimal control policy for a system one month after an outbreak. However, instead of implementing the policy matched to the actual epidemic age, we enforce the optimal control policy 30 days later, i.e. at the end of 60 days after the start of the outbreak. Figure S7 shows the difference in the mistimed control policy vs. the optimal control policy; as is evident the mistimed policy relaxes stringent lockdown when the optimal policy continues to lockdown. As a consequence the total deaths are far higher for $25 \%$ and $50 \%$ isolation efficiency (see Table 1). The mistimed policy, in effect, biases the system toward minimizing socioeconomic rather than public health costs. This significant difference in performance metrics demonstrates the potential shortcomings of implementing a policy based on optimal control. However, we note that with a stringent isolation efficiency, delays are less problematic. The reason is that with efficient infected case isolation, both the mistimed and optimal control policy could enable nearly all individuals to work, given that $\mathcal{R}_{\text {eff }}$ is held below 1 by infection isolation on its own.

Despite its fragility, we identify common features of the optimal control policy given variation in the effectiveness of infectious case isolation. First, the optimal control policy minimizes infected contact rates. The optimal control solutions also robustly identify an immune shielding strategy such that recovered/immune individuals elevate their interactions to the maximum possible relative to baseline. Importantly, differences in the optimal control policy are primarily centered on identifying a switch point in contact rate level for the susceptible population. From Figures S4-S6, we observe the switch point as a function of time, showing that irrespective of isolated case effectiveness and shield immunity constraints, the increase in susceptible contact rates happens later in the lockdown period. Switchover points correspond to times when the infection prevalence is relatively low compared to the recovered/immune population. This observation provides the basis for a feedback, rather than optimal, control policy.

We propose the use of a feedback control policy adapted from emergent features of the optimal control policy solutions: (i) infectious individuals isolate as far as is possible; (ii) recovered/immune individuals increase their activities as much as possible, i.e., akin to shield immunity. Hence, we set out to identify a system-dependent change in the contact rate of susceptible individuals, separating lockdowns vs. return-towork. In practice, we identify a critical curve in $I-R$ plane (i.e., infected-recovered cases plane) via a genetic algorithm, such that the recommended behavior of susceptible individuals is dictated by surveillancebased estimates of infectious and recovered/immune individuals (see supplemental information for more details).

Figure 4 summarizes the results of the feedback control policy. From a policy perspective, the feedback control policy identifies a switch between lockdown and return-to-work when there are significantly more recovered/immune individuals than infectious individuals. The timing of the return from lockdowns is accelerated given increases in isolation efficiency (rows) with additional benefits from the implementation of shield immunity (columns). Typically, the transition between lockdown and re-openings occur when circulating case levels are low relative to recovered/immune individuals. The critical ratio of recovered to infectious individuals decreases as isolation increases. Notably, when there are sufficiently high 
Lockdown 1x Open

$35 \%$

Isolation

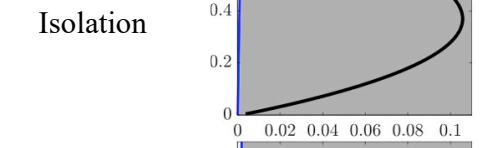

$45 \%$

Isolation

$65 \%$

Isolation

$75 \%$

Isolation
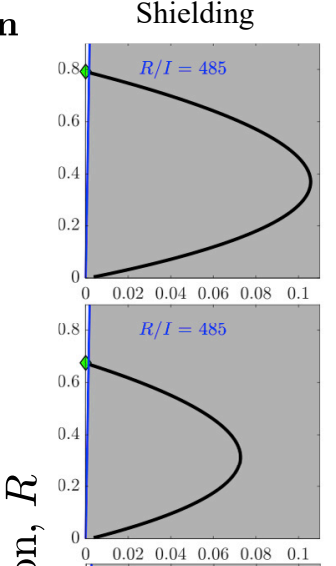

$55 \%$

Isolation

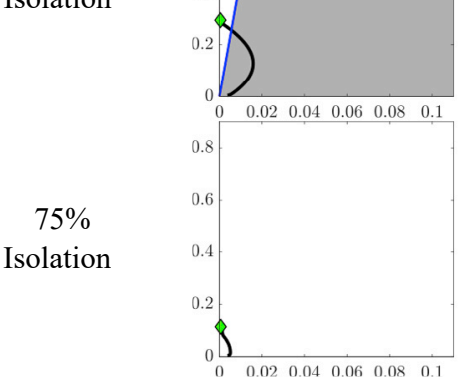

2x

Shielding
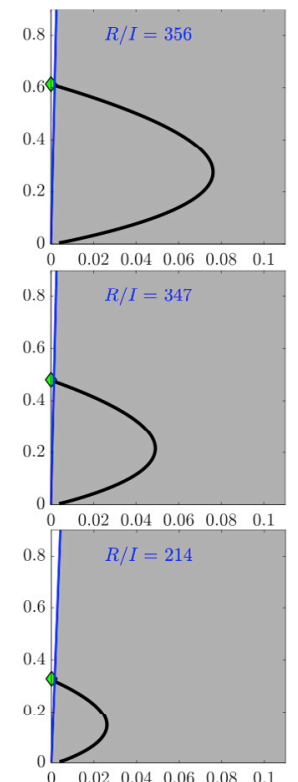

$\begin{array}{lllllll}0 & 0.02 & 0.04 & 0.06 & 0.08 & 0.1\end{array}$

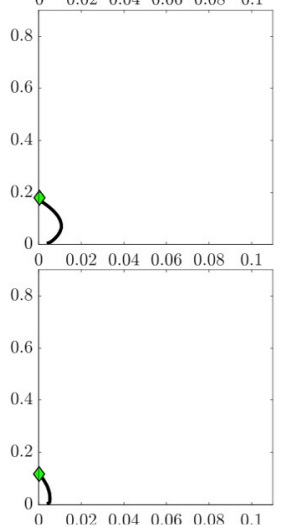

$4 \mathrm{x}$

Shielding
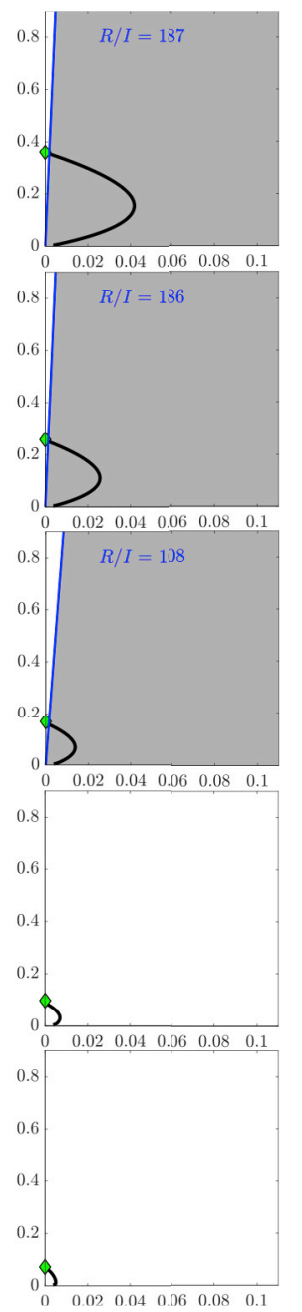

\section{Infected fraction, $I$}

Figure 4. Heuristic state feedback intervention policies varying with isolation efficiency (rows) and shielding levels (columns)

In each panel, the trajectory is noted in black with the final state as green diamond. An optimal line divides the plane into two regions which determines the optimal contact rate for the susceptible population for the current infected and recovered cases. The optimal policy in the dark gray region is lockdown and open in the white region. The phase plots show the dynamics of the infected and recovered case fractions over the period of 360 days, while applying the control strategy described above in the absence of shielding and for shielding levels of 2 and 4 respectively. For the case of isolation efficiency of $75 \%$, no lockdown is needed at all for the susceptible population. See supplemental information for a larger set of plots with increments of $5 \%$ in isolation efficiency and for shielding levels of 2, 3, 4, and 5 .

levels of isolation of infectious individuals then the optimal feedback policy suggests that no lockdown is required (note the entirely 'white' regions in the bottom row). In the discussion section, we provide additional context on the potential impacts of vaccination on this central finding.

Critically, the performance of the test-driven feedback policy is nearly identical for the performance metrics with or without mistiming (see Table 2). This finding implies that state-based approaches will be less likely to have exponentially mistimed applications, and reinforces the need for population-scale testing for both active infections and recovered/immune individuals. To examine the robustness of the feedback strategy in terms of model mis-specification, we consider three cases in which the isolation efficiency is unbiased, 


\begin{tabular}{|c|c|c|c|c|}
\hline \multirow{2}{*}{$\begin{array}{l}\text { Feedback control approach } \\
\text { Efficiency of } \\
\text { isolation } \\
\text { (shielding }=2 \text { ) }\end{array}$} & \multicolumn{2}{|l|}{ Without delay } & \multicolumn{2}{|l|}{ With delay } \\
\hline & $\begin{array}{l}\text { Total deaths } \\
\text { (per } 100,000 \\
\text { individuals) }\end{array}$ & $\begin{array}{l}\text { Working } \\
\text { fraction }\end{array}$ & $\begin{array}{l}\text { Total deaths } \\
\text { (per 100,000 } \\
\text { individuals) }\end{array}$ & $\begin{array}{l}\text { Working } \\
\text { fraction }\end{array}$ \\
\hline $25 \%$ & 620 & $89.98 \%$ & 620 & $89.94 \%$ \\
\hline $50 \%$ & 250 & $62.40 \%$ & 250 & $62.12 \%$ \\
\hline $75 \%$ & 30 & $99.90 \%$ & 30 & $99.90 \%$ \\
\hline
\end{tabular}

The comparisons are made for isolation efficiencies of $25 \%, 50 \%$ and $75 \%$, with performance metrics of total deaths and working fraction. Both performance metrics are nearly identical for all efficiencies, suggesting no significant effect of delay on the system.

overestimated (by $\sim 10 \%$ ) and underestimated (by $\sim 10 \%$ ) relative to the true value. We then compare the total deaths and working fraction of a feedback strategy based on these (potentially incorrect) estimates of the isolation efficiency. We find that the feedback strategy is robust to such mis-specification, particularly when isolation efficiency is high or low (see Figure S11 for details). We also note that a simple policy with only two states - "lockdown" and "open", respectively, corresponding to minimum and baseline contact rates for the susceptible cases, would be easier to implement than one with continuous "phases" or state changes. In Figures S9 and S10, we document the generalizability of results given variation in infected case isolation and the level of shield immunity.

\section{DISCUSSION}

We have developed a linked series of optimal and feedback control analyses to evaluate the effectiveness (and benefits) of modifying contact rates for managing the COVID-19 pandemic from both health and economic perspectives. Throughout, our central goal was to optimize the interactions between individuals based on disease status so as to achieve a defined balance between public health and economic outcomes. By explicitly incorporating contact rates as the control variables in an SEIR model, we were able to identify optimal control policies that could, in theory, significantly reduce expected infections (and fatalities) while reducing the negative socioeconomic costs of sustained lockdowns. Optimal control policies are unlikely to be applied in practice, given the potential exponential mis-specification of policies over time. Hence, we leveraged insights from the optimal control solutions to guide a feedback control approach that performs nearly, as well as the optimal control approach with significant improvements in robustness given uncertainty in estimating the epidemic state. Collectively, our control policies indicate that infected individuals should be isolated (as effectively as possible), recovered/immune individuals should be encouraged to return-to-work (given benefits accrued via shield immunity), while the release of other individuals from lockdown should be guided by the epidemic state. The transition from lockdown to return-to-work occurs when circulating case-loads are far lower than recovered case counts; with the scope of the epidemic sharply controlled by infection case isolation. A combination of policies, e.g., mask-wearing, physical distancing, will help to reduce transmission risk for individuals who do return-to-work.

A salient point that emerged from the control analysis is the benefit of reducing interactions by infectious individuals and increasing interactions by recovered/immune individuals. In doing so, it is critical to note that the conception of the model preceded the availability of vaccines. The increase of activity by recovered/immune individuals effectively dilutes risky contacts between susceptible and infectious individuals. We contend that this principle of shield immunity is also relevant when individuals are vaccinated, and therefore move from susceptible directly to the recovered/immune category. Increases in vaccination may provide opportunities to reduce risk for susceptible individuals, beyond benefits accrued by susceptible depletion alone. We recognize that adopting policies that include individual disease status are likely to raise both privacy and ethical concerns (Phelan, 2020; Norheim, 2020). Yet, given the slow rate of vaccine dissemination, we suggest that the absence of action-taking that could increase protection to those yet to be vaccinated also comes at a public health and socioeconomic cost. Even now, more than a year after the identification of the first SARS-CoV-2 case, we remain closer to the beginning than the end of the Covid-19 pandemic. As we have shown, accelerating the slowdown of transmission while restoring economic activity may be enabled by both personalized, test-driven, policies as the basis for mitigation that reduce risk for all. 


\section{Limitations of the study}

The SEIR framework used as the basis for the present control study is intentionally simplified. The epidemic model does not account for process or observational noise, analytic test features, heterogeneity, stratified risk, asymptomatic cases, and detailed elaboration of severe cases. By reducing the model complexity, we have tried to shed light on the general problem of balancing public health with socioeconomic outcomes. In doing so, we have highlighted a middle ground between dichotomous outcomes that focus on public health or socioeconomic costs solely. We recognize that extensions and applications of the present work will require consideration of additional epidemic complexities (e.g., asymptomatic transmission) and additional evaluation of joint public health and economic costs (e.g., arising from hospitalization burden). Translating the present findings into practical use will also require improved assessments of the ways in which behavior is influenced by awareness and communication of the pandemic state (Weitz et al., 2020b; Franco, 2020), in addition to evaluating the influences of formal implementation of policy campaigns.

\section{STAR $\star$ METHODS}

Detailed methods are provided in the online version of this paper and include the following:

- KEY RESOURCES TABLE

- RESOURCE AVAILABILITY

O Lead contact

Materials availability

Data and code availability

- METHOD DETAILS

\section{SUPPLEMENTAL INFORMATION}

Supplemental information can be found online at https://doi.org/10.1016/j.isci.2021.102710.

\section{ACKNOWLEDGMENTS}

We thank Weitz Group team members for comments and feedback. This work was supported by grants from the Army Research Office (W911NF1910384), National Institutes of Health (1R01A146592-01) and the National Science Foundation (1806606 and 2032082). The sponsor of the study had no role in study design, data analysis, results interpretation, or writing of the report. All corresponding authors had full access to all the data in the study and shared the final responsibility for the decision to submit for publication.

\section{AUTHOR CONTRIBUTIONS}

J.S.W. and M.E.H. conceived and designed the study, including epidemiological framework. G.L., S.S., and Y.W. developed the core modeling and control frameworks, developed numerical algorithms. G.L. and S.S. developed simulation code and performed computational analysis. All authors contributed to writing and editing of the article. All authors read and approved the final article.

\section{DECLARATION OF INTERESTS}

The authors declare no competing interests.

Received: January 25, 2021

Revised: April 13, 2021

Accepted: June 8, 2021

Published: July 23, 2021

\section{SUPPORTING CITATIONS}

The following reference appears in the Supplemental information: Anderson and May, 1992; Kirk, 2004; Liberzon, 2011; Ngonghala et al., 2020; Polak, 2012; The MathWorks, 2020; Whitley, 1994. 


\section{REFERENCES}

Amanat, F., Stadlbauer, D., Strohmeier, S., Nguyen, T.H.O., Chromikova, V., McMahon, M., Jiang, K., Arunkumar, G.A., Jurczyszak, D., Polanco, J., et al. (2020). A serological assay to detect SARS-CoV-2 seroconversion in humans. Nat. Med. 26, 1033-1036. https://doi.org/10. 1101/2020.03.17.20037713.

Anderson, R.M., and May, R.M. (1992). Infectious Diseases of Humans: Dynamics and Control (Oxford University Press).

Atkeson, A., Droste, M.C., Mina, M., and Stock, J.H. (2020). Economic Benefits of Covid-19 Screening Tests (No. W28031) (National Bureau of Economic Research). https://doi.org/10.3386/w28031.

Baqaee, D., Farhi, E., Mina, M.J., and Stock, J.H. (2020). Reopening Scenarios (No. W27244)

(National Bureau of Economic Research). https:// doi.org/10.3386/w27244.

Bodkin, C., Mokashi, V., Beal, K., Wiwcharuk, J., Lennox, R., Guenter, D., Smieja, M., and O'Shea, T. (2020). Pandemic Planning in Homeless Shelters: a pilot study of a COVID-19 testing and support program to mitigate the risk of COVID-19 outbreaks in congregate settings. Clin. Infect. Dis. 72, 1639-1641. https://doi.org/ 10.1093/cid/ciaa743.

Bonnans, J.F., and Gianatti, J. (2020). Optimal Control Techniques Based on Infection Age for the Study of the COVID-19 Epidemic. https://hal. inria.fr/hal-02558980/document.

Brynjolfsson, E., Horton, J., Ozimek, A., Rock, D., Sharma, G., and TuYe, H.-Y. (2020). COVID-19 and Remote Work: An Early Look at US Data. https://doi.org/10.3386/w27344.

Cheng, V.C.-C., Wong, S.-C., Chuang, V.W.-M., So, S.Y.-C., Chen, J.H.-K., Sridhar, S., To, K.K.W. Chan, J.F.W., Hung, I.F.N., Ho, P.L., and Yuen, K.Y. (2020). The role of community-wide wearing of face mask for control of coronavirus disease 2019 (COVID-19) epidemic due to SARS-CoV-2 J. Infect. 81, 107-114.

Chinazzi, M., Davis, J.T., Ajelli, M., Gioannini, C., Litvinova, M., Merler, S., Pastore y Piontti, A., Mu, K., Rossi, L., Sun, K., et al. (2020). The effect of travel restrictions on the spread of the 2019 novel coronavirus (COVID-19) outbreak. Science 368, 395-400.

Chu, D.K., Akl, E.A., Duda, S., Solo, K., Yaacoub, S., Schünemann, H.J., El-harakeh, A., Bognanni, A., Lotfi, T., Loeb, M., et al. (2020). Physical distancing, face masks, and eye protection to prevent person-to-person transmission of SARSCoV-2 and COVID-19: a systematic review and meta-analysis. Lancet 395, 1973-1987.

Coglianese, C., and Mahboubi, N.A. (2021). Administrative Law in a Time of Crisis: Comparing National Responses to COVID-19. http://www. administrativelawreview.org/wp-content/ uploads/2021/03/07.-ALR-73.

1 Foreward_FINAL.pdf.

Cowling, B.J., Ali, S.T., Ng, T.W.Y., Tsang, T.K., Li, J.C.M., Fong, M.W., Liao, Q., Kwan, M.Y., Lee, S.L., Chiu, S.S., et al. (2020). Impact assessment of non-pharmaceutical interventions against coronavirus disease 2019 and influenza in Hong Kong: an observational study. Lancet Public
Health 5, e279-e288. https://doi.org/10.1016/ s2468-2667(20)30090-6.

Denny, T.N. (2020). Implementation of a pooled surveillance testing program for asymptomatic sars-cov-2 infections on a college campus-Duke university, durham, north carolina, august 2october 11, 2020. MMWR. Morbidity and Mortality Weekly Report, 69. https://doi.org/10. 15585/mmwr.mm6946e1.

Douglas, M., Katikireddi, S.V., Taulbut, M. McKee, M., and McCartney, G. (2020). Mitigating the wider health effects of covid-19 pandemic response. BMJ 369, m1557.

Fauci, A.S., Lane, H.C., and Redfield, R.R. (2020). Covid-19 - navigating the uncharted. N. Engl. J. Med. 382, 1268-1269.

Feng, S., Shen, C., Xia, N., Song, W., Fan, M., and Cowling, B.J. (2020). Rational use of face masks in the COVID-19 pandemic. Lancet Respir. Med. 8, 434-436.

Ferguson, N., Laydon, D., Nedjati Gilani, G., Imai, N., Ainslie, K., Baguelin, M., Bhatia, S., Boonyasiri, A., Cucunuba Perez, Z., Cuomo-Dannenburg, G. et al. (2020). Report 9: Impact of Nonpharmaceutical Interventions (NPIs) to Reduce COVID19 Mortality and Healthcare Demand. http://hdl.handle.net/10044/1/77482.

Ferretti, L., Wymant, C., Kendall, M., Zhao, L., Nurtay, A., Abeler-Dörner, L., Parker, M., Bonsall, D., and Fraser, C. (2020). Quantifying SARS-CoV-2 transmission suggests epidemic control with digital contact tracing. Science 368. https://doi.org/10. 1126/science.abb6936.

Flaxman, S., Mishra, S., Gandy, A., Unwin, H.J.T. Mellan, T.A., Coupland, H., Whittaker, C., Zhu, H., Berah, T., Eaton, J.W., et al. (2020). Estimating the effects of non-pharmaceutical interventions on COVID-19 in Europe. Nature 584, 257-261. https://doi.org/10.1038/s41586-020-2405-7.

Franco, E. (2020). A feedback SIR (fSIR) model highlights advantages and limitations of infection-dependent mitigation strategies. arXiv. https://arxiv.org/abs/2004.13216.

Gibson, G., Weitz, J.S., Shannon, M.P., Holton, B., Bryksin, A., Liu, B., Bramblett, S., Williamson, J., Farrell, M., Ortiz, A., et al. (2021). Surveillance-todiagnostic testing program for asymptomatic SARS-CoV-2 infections on a large, urban campusGeorgia institute of technology, fall 2020. MedRxiv. https://doi.org/10.1101/2021.01.28.21250700.

Greenhalgh, T., Schmid, M.B., Czypionka, T., Bassler, D., and Gruer, L. (2020). Face masks for the public during the covid-19 crisis. BMJ 369 m1435.

He, X., Lau, E.H.Y., Wu, P., Deng, X., Wang, J. Hao, X., Lau, Y.C., Wong, J.Y., Guan, Y., Tan, X., et al. (2020). Temporal dynamics in viral shedding and transmissibility of COVID-19. Nat. Med. 26, 672-675. https://doi.org/10.1038/s41591-020 0869-5.

Hochberg, M.E. (2020). Importance of suppression and mitigation measures in managing COVID-19 outbreaks. https://doi.org/ 10.1101/2020.03.31.20048835.
Hsiang, S., Allen, D., Annan-Phan, S., Bell, K., Bolliger, I., Chong, T., Druckenmiller, H., Huang, L.Y., Hultgren, A., Krasovich, E., et al. (2020). The effect of large-scale anti-contagion policies on the COVID-19 pandemic. Nature 584, 262-267.

Kirk, D.E. (2004). Optimal Control Theory: An Introduction (Courier Corporation).

Kraay, A.N.M., Nelson, K., Zhao, C., Weitz, J.S. and Lopman, B.A. (2020). Modeling serological testing to inform relaxation of social distancing for COVID-19 control. medRxiv. https://doi.org/ 10.1101/2020.04.24.20078576.

Kraemer, M.U.G., Yang, C.-H., Gutierrez, B., Wu, C.-H., Klein, B., Pigott, D.M., Du Plessis, L., Faria N.R. Li, R. Hanage, W.P. et al. (2020). The effect of human mobility and control measures on the COVID-19 epidemic in China. Science 368, 493-497.

Krammer, F., and Simon, V. (2020). Serology assays to manage COVID-19. Science 368, 1060 1061.

Larremore, D.B., Wilder, B., Lester, E., Shehata, S., Burke, J.M. Hay, J.A. Tambe, M. Mina, M.J. and Parker, R. (2021). Test sensitivity is secondary to frequency and turnaround time for COVID-19 surveillance. Sci. Adv. 7, eabd5393.

Liberzon, D. (2011). Calculus of Variations and Optimal Control Theory: A Concise Introduction (Princeton University Press).

Morris, D. H Rossine, FW Plotkin, J.B and Levin, S.A. (2021). Optimal, near-optimal, and robust epidemic control. Commun. Phys. 4, article number 78 .

Ngonghala, C.N., Iboi, E., Eikenberry, S., Scotch, M. Maclntyre, C.R., Bonds, M.H., and Gumel A.B. (2020). Mathematical assessment of the impact of non-pharmaceutical interventions on curtailing the 2019 novel Coronavirus. Math. Biosci. 325, 108364

Norheim, O.F. (2020). Protecting the population with immune individuals. Nat. Med. 26, 823-824. https://doi.org/10.1038/s41591-020-0896-2.

OECD. (2020a). The territorial impact of COVID19: managing the crisis across levels of government. OECD Policy Responses to Coronavirus (COVID-19) [Internet]. https://read. oecd-ilibrary.org/view/?ref=128_128287 5agkkojaaa\&title=The-territorial-impact-ofcovid-19-managing-the-crisis-across-levels-ofgovernment.

OECD. (2020b). Flattening the unemployment curve policies to support workers' income and promote a speedy labour market recovery. OECD Policy Responses to Coronavirus (COVID-19) [Internet]. https://read.oecd-ilibrary.org/view/? ref=134_134515-cww8xuoucy\&title=Flatteningthe-unemployment-curve-Policies-to-supportworkers-income-and-promote-a-speedy-labourmarket-recovery.

Phelan, A.L. (2020). COVID-19 immunity passports and vaccination certificates: scientific equitable, and legal challenges. Lancet 395, 1595-1598. https://doi.org/10.1016/s01406736(20)31034-5. 
E. Polak, ed. (2012). In Optimization: Algorithms and Consistent Approximations, Vol. 124

(Springer, Science \& Business Media), pp. 482-645.

Specht, I., Sani, K., Botti-Lodovico, Y., Hughes, M., Heumann, K., Bronson, A., Marshall, J., Baron, E., Parrie, E., Glennon, O., et al. (2021). The case for altruism in institutional diagnostic testing. MedRxiv. https://doi.org/10.1101/2021.03.16. 21253669.

The MathWorks, I. (2020). Global Optimization Toolbox.

Walker, P.G.T. Whittaker, C. Watson, O.J. Baguelin, M., Winskill, P., Hamlet, A., Djafaara, B.A., Cucunubá, Z., Mesa, D.O., Green, W., et al. (2020). The impact of COVID-19 and strategies for mitigation and suppression in low- and middleincome countries. Science 369, 413-422.
Weitz, J.S., Beckett, S.J., Coenen, A.R., Demory, D., Dominguez-Mirazo, M., Dushoff, J., Leung, C.Y., Li, G., Măgălie, A., Park, S.W., et al. (2020a). Modeling shield immunity to reduce COVID-19 epidemic spread. Nat. Med. 26, 849-854.

Weitz, J.S., Park, S.W., Eksin, C., and Dushoff, J. (2020b). Awareness-driven behavior changes can shift the shape of epidemics away from peaks and toward plateaus, shoulders, and oscillations. Proc. Natl. Acad. Sci. U S A 17, 32764-32771.

Wells, C.R., Sah, P., Moghadas, S.M., Pandey, A., Shoukat, A., Wang, Y., Wang, Z., Meyers, L.A. Singer, B.H., and Galvani, A.P. (2020). Impact of international travel and border control measures on the global spread of the novel 2019 coronavirus outbreak. Proc. Natl. Acad. Sci. U S A 117, 7504 7509. https://doi.org/10.1073/pnas.2002616117.

Whitley, D. (1994). A genetic algorithm tutorial. Stat. Comput. 4, 65-85.
World Health Organization. (2021). COVID-19 Weekly Epidemiological Update.

Yong, S.E.F., Anderson, D.E., Wei, W.E., Pang, J., Chia, W.N., Tan, C.W., Teoh, Y.L., Rajendram, P., Toh, M.P.H.S., Poh, C., et al. (2020). Connecting clusters of COVID-19: an epidemiological and serological investigation. Lancet Infect. Dis. 20, 809-815.

Zhang, R., Li, Y., Zhang, A.L., Wang, Y., and Molina, M.J. (2020). Identifying airborne transmission as the dominant route for the spread of COVID-19. Proc. Natl. Acad. Sci. U S A 117 14857-14863.

Zhao, H., and Feng, Z. (2020). Staggered release policies for COVID-19 control: costs and benefits of relaxing restrictions by age and risk. Math. Biosci. 326, 108405. 


\section{STAR $\star$ METHODS}

\section{KEY RESOURCES TABLE}

\begin{tabular}{lll}
\hline REAGENT or RESOURCE & SOURCE & IDENTIFIER \\
\hline Software and algorithms & & \\
\hline Genetic Algorithm in Global Optimization & MathWorks, Natick MA & https://www.mathworks.com/products/ \\
$\begin{array}{l}\text { Toolbox, MATLAB R2019a } \\
\text { A MATLAB implementation of our dynamic }\end{array}$ & This paper & global-optimization.html \\
model & & https://github.com/WeitzGroup/ \\
\hline
\end{tabular}

\section{RESOURCE AVAILABILITY}

\section{Lead contact}

Further information and requests should be directed to and will be fulfilled by the lead contact Joshua S. Weitz (jsweitz@gatech.edu).

\section{Materials availability}

This study did not generate new unique reagents.

Data and code availability

All simulation and figure codes used in the development of this manuscript are available at https://github. com/WeitzGroup/COVID_Control_2020.

\section{METHOD DETAILS}

Please find method details in the file "Supplemental procedures". 